\title{
Active learning in optics and photonics: Fraunhofer diffraction
}

H. Ghalila, Z. Ben Lakhdar, S. Lahmar, Z. Dhouaidi, Y. Majdi

H. Ghalila, Z. Ben Lakhdar, S. Lahmar, Z. Dhouaidi, Y. Majdi, "Active learning in optics and photonics: Fraunhofer diffraction," Proc. SPIE 9289, 12th Education and Training in Optics and Photonics Conference, 92892V (17 July 2014); doi: 10.1117/12.2070776

Event: 12th Education and Training in Optics and Photonics Conference, 2013, Porto, Portugal 


\title{
Active Learning in Optics and Photonics: Fraunhofer diffraction

\author{
H. Ghalila ${ }^{1,2}$, Z. Ben Lakhdar ${ }^{1,2}$, S. Lahmar ${ }^{1,2}$, Z. Dhouaidi ${ }^{1,2}$, Y. Majdi ${ }^{1,2}$ \\ ${ }^{2}$ Société Tunisienne d'Optique
} \\ ${ }^{1}$ Departement of physics-Faculty of Sciences - University Tunis elManar-Tunisia
}

\begin{abstract}
"Active Learning in Optics and Photonics" (ALOP), funded by UNESCO within its Physics Program framework with the support of ICTP (Abdus Salam International Centre for Theoretical Physics) and SPIE (Society of Photo-Optical Instrumentation Engineers), aimed to helps and promotes a friendly and interactive method in teaching optics using simple and inexpensive equipment. Many workshops were organized since 2005 the year when Z. BenLakhdar, whom is part of the creators of ALOP, proposed this project to STO (Société Tunisienne d'Optique). These workshops address several issues in optics, covering geometrical optics, wave optics, optical communication and they are dedicated to both teachers and students. We focus this lecture on Fraunhofer diffraction emphasizing the facility to achieve this mechanism in classroom, using small laser and operating a slit in a sheet of paper. We accompany this demonstration using mobile phone and numerical modeling to assist in the analysis of the diffraction pattern figure.
\end{abstract}

Keywords: Fraunhofer diffraction, active learning, low cost apparatus, numerical modeling, mobile phone.

\section{Introduction}

Nothing that we see in our daily live can escape diffraction and yet it remains difficult to explain to students. Workshops organized within the framework of the project "Active Learning in Optics and Photonics" (ALOP) mainly funded by UNESCO, with the support of ICTP (Abdus Salam International Centre for Theoretical Physics) and SPIE (Society of Photo-Optical Instrumentation Engineers) aim to make simpler the introduction of this phenomenon and to accompany the classical mathematical approach [1-5]. This ALOP project consists in a series of workshops for educators, researchers and students and promotes an innovative method of teaching physics using simple, inexpensive material that can be fabricated locally. The main idea of these workshops is based on the use of homemade material and hands-on activities in the classroom. A special care is given to Predictions, Observations, Discussions and Syntheses (PODS), in order to refine the interpretation of these experiments. ALOP workshops offer high-school college teachers and university physics teachers the opportunity to improve their conceptual understanding of optics. Fifteen workshops have been organized in Tunisia since 2005. These workshops usually run for three or five days depending on the number of modules studied and with classes of twenty or thirty participants. Lessons deal with several topics usually found in any introductory university physics program as geometrical optics, interference and diffraction, atmospheric optics, optics in communications $[1,6]$.

In this paper, we will focus on Fraunhofer diffraction and interference pattern obtained by two slits. The experimental set-up was already used for several national and regional ALOP workshops [6]. In addition to the specific hands-on ALOP activities, we propose to use the mobile phone and also numerical modeling as complements for analyzes. Nowadays, taking pictures with cell phone is almost a reflex and in a way this doesn't alter the spirit of the ALOP approach (PODS). Unlike the cell phone, numerical modeling remains an abstract and opaque concept for a wide majority of students but it is a strong link between mathematical models and experimental observations. In this paper, we present a simple way to accomplish the link between observations of the diffraction-interference pattern obtained by a low cost apparatus and the mathematical functions. At the same time we show a way to reach back the width of the handmade slits or to check out the width of light spots in the diffraction pattern figure. 


\section{Experimental execution}

Among the six modules taught by ALOP, the module 3 named "interference and diffraction" [6] may be the easiest lesson to achieve experimentally because it needs an inexpensive material easy to make and very accessible all over the world. This material can be resumed in a laser pointer which cost less than $1 \$$ in many countries and a slit operated in a sheet of paper or in a backside of mirror by cutter or a very sharp object as it is shown in figure.1. The wall of the classroom acts as the screen for the observation of the light pattern.

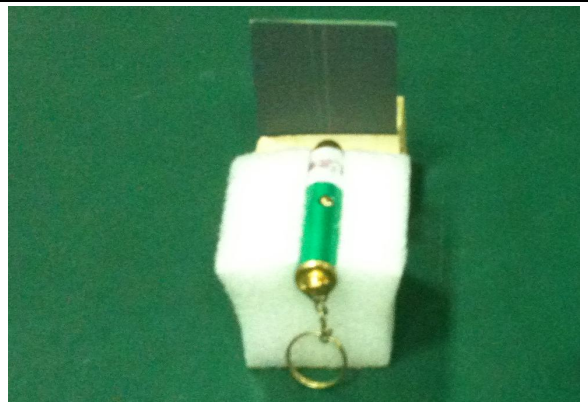

a)

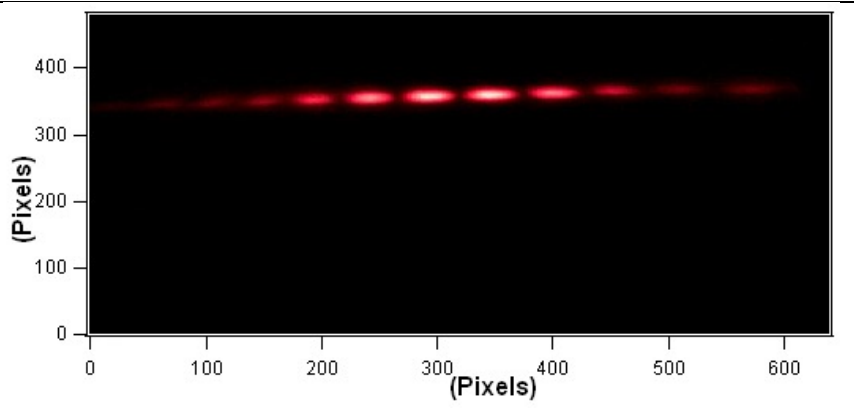

b)

Figure.1 : a) Laser pointer fixed on a small patch of polystyrene ahead slits made by cutter on a backside of mirror. b) Diffraction-interference pattern figure observed at $5 \mathrm{~m}$ from the mirror with a $635 \mathrm{~nm}$ wavelength.

The challenge in this module is to apply the PODS philosophy by testing different configurations of slits (shape and number) operated manually on any kind of support and also by changing various parameters as: wavelength, distance of the screen, angle of incidence, etc. For instance, figure.1-b) shows the diffraction pattern observed on a screen positioned at $5 \mathrm{~m}$ from the mirror by a laser pointer operating at $635 \mathrm{~nm}$. The question we can ask here is what will be the change on this pattern if we increase the distance between the mirror and the screen. In this case, PODS is easily applied because it is simple to change this distance and confront predictions with observations. Another question we can investigate is what will be the impact of the decrease of the wavelength. This example is interesting, because it is more complicated to change the incident wavelength, laser-pointers are not available for all wavelength, and, as we will see in chapter.3, cell phones and numerical modeling can help to apply PODS in this case by the same way we would if we had to change another parameter.

\section{Cell phones and numerical modeling}

Nowadays, it is relatively easy to find in most developing countries cell phones equipped with camera and generally most of the participants ALOP have ones. This is a good point because it is very interesting to capture the multiple figures we scan during a session for further analyses. The image shown in figure.1-b) is an example for this. The accessibility to computer remains more difficult but its expansion is incredibly fast. So we can hope that this facility will be available very soon for all teachers and students all over the world.

\subsection{Image analysis}

As a preparation to future ALOP session, we tested different slits made with cutter on backside of mirror and for each one we captured the light pattern projected on the wall of the classroom in order to check the feasibility and to compare between the various images. With the help of software dedicate to the handling of the image, it is possible to represent the intensity variation along the diffraction pattern in a specific direction. We use here the Igor Pro version 6 software that works on a computer. Figure.2-b) illustrate the intensity variation versus the number of pixel for the case described in figure.1-b). To get this with Igor Pro, we use the profile tool afforded by this software and simply draw a straight line (white dashed line on figure.2-a)) through the entire diffraction pattern. 


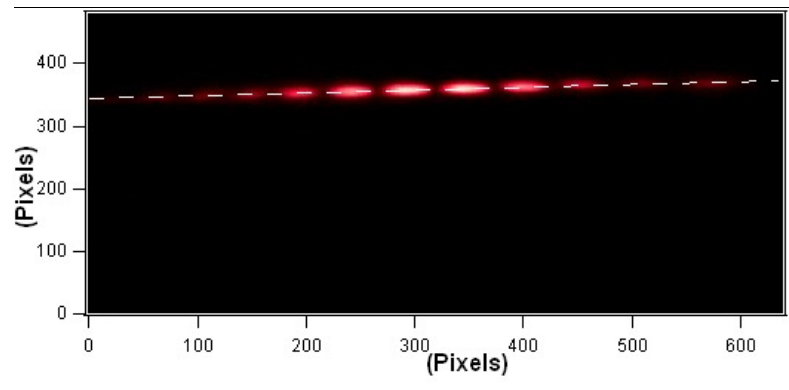

a)

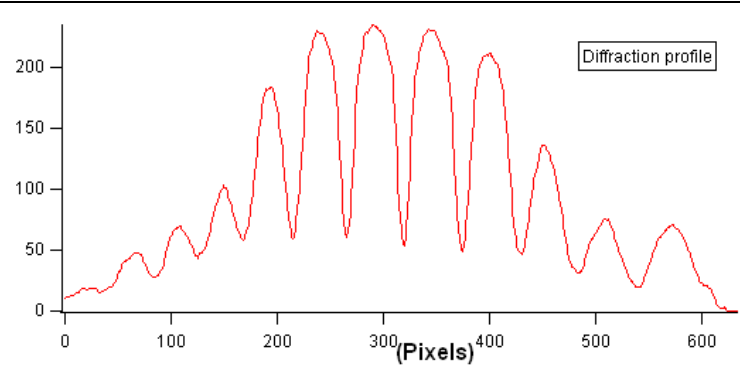

b)

Figure.2: Drawing line profile along the diffraction pattern a) automatically generate the variation intensity b).

A fast observation at the intensity variations (figure.2-b)) shows a slow oscillation modulated by a fast one. For an expert eye, it is easy to guess that the slow one corresponds to the module of sinus cardinal and the fast one to the module of cosine function. But, it is not possible at this stage to define the mathematical function that will fit this behavior. The question that arises is how can we link simply this theoretical prediction with our observation.

\subsection{Numerical modeling}

As we can see in figure.2-b), the abscissa values designate the number of the pixel and not the real space. The values in vertical axis express the relative variation of the intensity in arbitrary units. We now from the wave theory of light the mathematical expression of double-slit experiment. In a normalized form, the expression given bellow renders the relative variation of the intensity avoiding in this way the impact of many unwanted fluctuations. The comparison between figure.2-b) and figure.3 shows a very similar behavior and this observation suggest that, with some adjustments of the parameters of the function $I(x)$, it will be possible to fit the experimental diffraction pattern. The major difference between these two curves is the transition from pixel space to the real physical space.

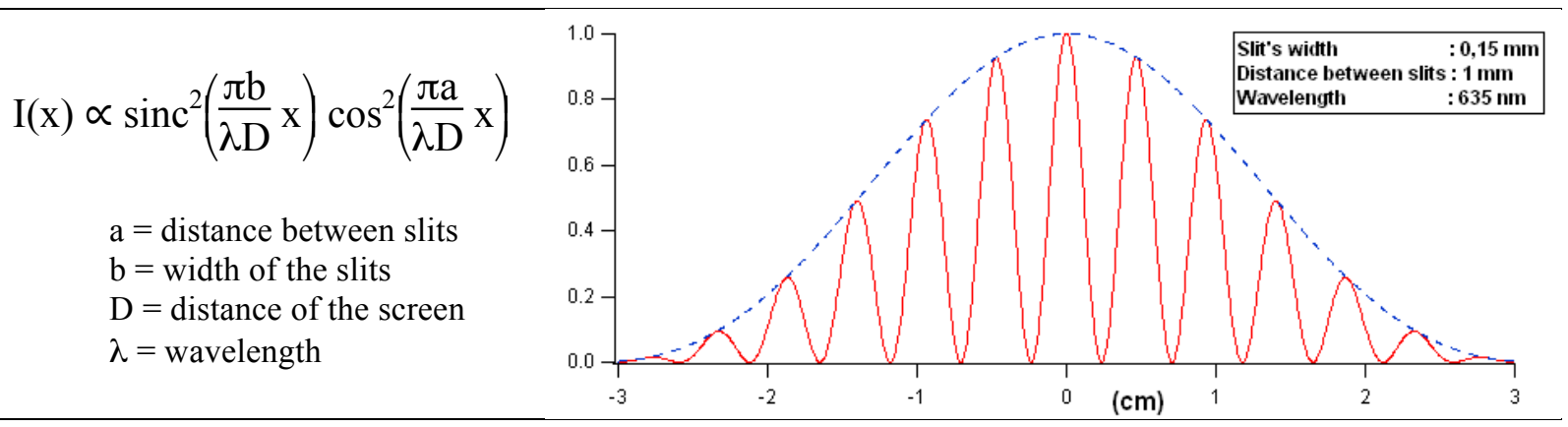

Figure.3 : Plots of the expression of $\mathrm{I}(\mathrm{x})$. The dashed line corresponds to the squared sinus cardinal and the solid line to the squared cosine function.

During the ALOP session some parameters are known and other measurable. Thus the wavelength of the laser pointer is known and in the present case $\lambda=635 \mathrm{~nm}$. The distance between the mirror and the screen (here the wall) is measurable with a ruler and in this case $\mathrm{D}=5 \mathrm{~m}$. For many configurations, the distance between the slits is measurable but not very accurate. In the case displayed here this distance is $\mathrm{a}=1 \mathrm{~mm}$. The only value, which is immeasurable at all with the cheap equipment used, is the width of the slit. To get it we can in one hand measure with a ruler the width of the spot of light observed in the screen. In the other hand we can adjust the parameter " $b$ ", in order to have the correspondence of this width with the abscissa value.

As I mentioned above, PODS are relevant for some parameters because they are easy to change. But, it is not possible to get a laser pointer for all wavelengths and then it becomes difficult to check prediction without observations. This example shows how much the use of the numerical modeling can be very 
convenient. The major problem with this is that we will be obliged to have computer on each table during the lessons and this will take us away from the philosophy of ALOP that is essentially experimental.

The needs for a computer can be avoided, if we implement this expression with useful interface on an app for a smartphone. In this way, it will be possible to measure very small objects (under the millimeter) with very simple and accessible equipment. Currently, the software we use to present the results in this paper works on computer and we are preparing a version that will work on the web and another one as smartphone app.

\section{Conclusion}

PODS philosophy is a very interesting approach to encourage the students to become aware of physical laws and also to improve them good physical reasoning skills. This approach will be optimized by multiplying the number of experiments achieved during the small period of time dedicated for this module. Frustration caused by the incapacity to test some predictions can be bypassed by the use of numerical modeling. Then, thanks to computers, we show how to explore trends and nuances by numerically

modifying some parameters. In order to remain in the spirit of ALOP, i.e. low cost and accessibility of the equipment, we plan to translate the actual software, which is working on computer, to smartphone app.

\section{REFERENCES}

[1] M. Alarcon, E. Arthurs, Z. Ben Lakhdar, I. Culaba, V. Lakshminarayanan, J. Maquiling, A. Mazzolini, J. Niemela, D. Sokoloff: "Active learning in optics and photonics: experiences in Africa”, ETOP 2005, http://spie.org/etop/ETOP2005_040.pdf

[2] J. Mazzolini : World Conference on Physics and Sustainable Development : UNESCO: Active Learning in Physics for Developing Countries of Asia \& Africa http://www.wcpsd.org/posters/education/Mazzolini.pdf

[3] Z. Ben Lakhdar, N. Derbel, Z. Dhaouadi, H. Ghalila, R. Miled, S. Lahmar, K. Berrada, R. Channa, A. Outzourhit : Active learning in physics a way for rational thinking - a way for development. http://spie.org/etop/2007/etop07programsI.pdf

[4] M. Zghal, H. Ghalila, Z. Ben Lakhdar: "A simple wavelength division multiplexing system for active learning teaching", ETOP 2009, http://spie.org/etop/2009/etop2009_10.8.60.pdf

[5] M. Alarcon, Z. Ben Lakhdar, I. Culaba, S. Lahmar, V. Lakshminarayanan, A. Mazzolini, J. Maquiling, Niemela J. " Active learning in optics and photonics (ALOP): a model for teacher training and professional development”, ETOP 2010, http://spie.org/x648.html?product_id $=860708$

[6] M. Alarcon, E. Arthurs, Z. Ben Lakhdar, I. Culaba, G. Denardo, V. Lakshminarayanan, J. Maquiling, A. Mazzolini, J. Niemela, D. Sokoloff: "Active learning in optics and photonics: Training Manual" http://unesdoc.unesco.org/images/0021/002171/217100e.pdf 